\title{
APPLICATION OF DATA LAKE APPROACH FOR DATA ANALYTICS IN E-HEALTH ISSUES OF ARCHITECTURES AND ORGANIZATION
}

\author{
Bálint Molnár ${ }^{1}$ and Ádám Tarcsi ${ }^{2}$ \\ ${ }^{I}$ Eötvös Loránd University, Budapest, Hungary \\ Department of Information Systems \\ ${ }^{2}$ Eötvös Loránd University Faculty of Informatics, \\ Data Science and Engineering Department \\ IoT\&Data Innovation Lab
}

\begin{abstract}
By a case study approach, the paper analyzes a project and information ecosystem of the Clinical Research environment within a Medical School at a University. There are two types of data sources, a medical information system based on a transactional database for recording patients and serving as the basis of nursing and treatments of patients and medical and other medical IoT systems (like ECGs, etc). There exists neither Data Warehouse solutions nor any other data collection solutions dedicated to utilizing Data Science methods and tools. The emerging technologies provide opportunities for synergy between the traditional Data Warehouse and the most recent Data Lake approaches. Therefore, it seems feasible and reasonable to integrate these two architecture approaches to support data analytics on the research of medicine and biomedical areas.
\end{abstract}

\section{KEYWORDS}

Data Warehouse, Data Lake, Enterprise Architecture, Data Science

\section{INTRODUCTION}

The healthcare and the healthcare industry is one of the major sources for generating huge data collections. In healthcare, the exploitation of data efficiently and effectively is a critical issue. Data analytics looks back a long history as the analysis of data is a long-practiced activity that started with descriptive statistics then, later on, continued with second-order statistics. The huge volume of data that are generated in Health-Care Information Systems comes from sources as Electronic Health Record, IoT (Internet of Things) devices monitoring physiologic states, medical pieces of equipment, genomic data, radiology, etc.

\section{DATA LAKE AND DATA WAREHOUSE}

Generally, organizations are full of data that are stored in existing databases, produced by various information systems, data streams are coming from mobile applications, social media, web information systems, and other devices that are linked to the internet (Internet of Things, IoT). This statement is true in an e-Health environment too as healthcare organizations cope with data that are gathered by information systems dedicated to patients' medical records, transcripts of audio records of Medical Doctors. The literature makes difference among Electronic Medical Record (EMR), Electronic Health Record (EHR), Personal Health Record (PHR), Computerized Provider Order Entry (CPOE), Picture Archiving and Communication System (PACS) (Shachak \& Reis, 2011) . The collected data can be considered as heterogeneous in both structure and content, i.e. there are structured, semi-structured, and unstructured data items, some of them are accompanied by meta-data. 
There is an essential difference regarding data capture in Business Information Systems and Information Systems of Healthcare. In a healthcare environment, data gathering is typically manual, and even the structured data contains plenty of textual information. The input data are created typically by human agents who can make errors in data either intentionally or unintentionally. Examples of error-prone data entry are as follows: name, address, identifiers (social security numbers). Thereby, the method of data entry leads to imprecise data that makes difficult any data mining exercises, e.g. association relationships that may have been found prove to be erroneous. While inputting data into the Information System of Electronic Health Record, the Medical Doctors frequently use either templates or copy-paste commands to fill in various forms as e.g. medical flow forms to comply with policies, standards, and other regulations. The problem with that practice is that it conceals the subtle differences among the electronic patients' records that would be precious for data analytics and knowledge discovery. Information that is produced by automated systems along with auto-fill and edit check options as e.g. speech to text, OCR (optical character recognition) that computerize patients' data may introduce systematic and random inaccuracies that differ from physicians to physicians, software tool to software tool. These errors are difficult to quantify and forestall. Moreover, there can be uncertainty in explanations of diagnosis and procedures among Medical Doctors, and then the encoding of semantic contents may differ as well.

To ensure, that there will be a centralized location that would serve as the single source of truth, the data with different types and structures from various sources should be collected and loaded into a Data Lake. The most recent technologies can yield opportunities for the application of data analytics and models of Data Science. The results of running data analytic algorithms could be actionable knowledge in the clinical research environment.

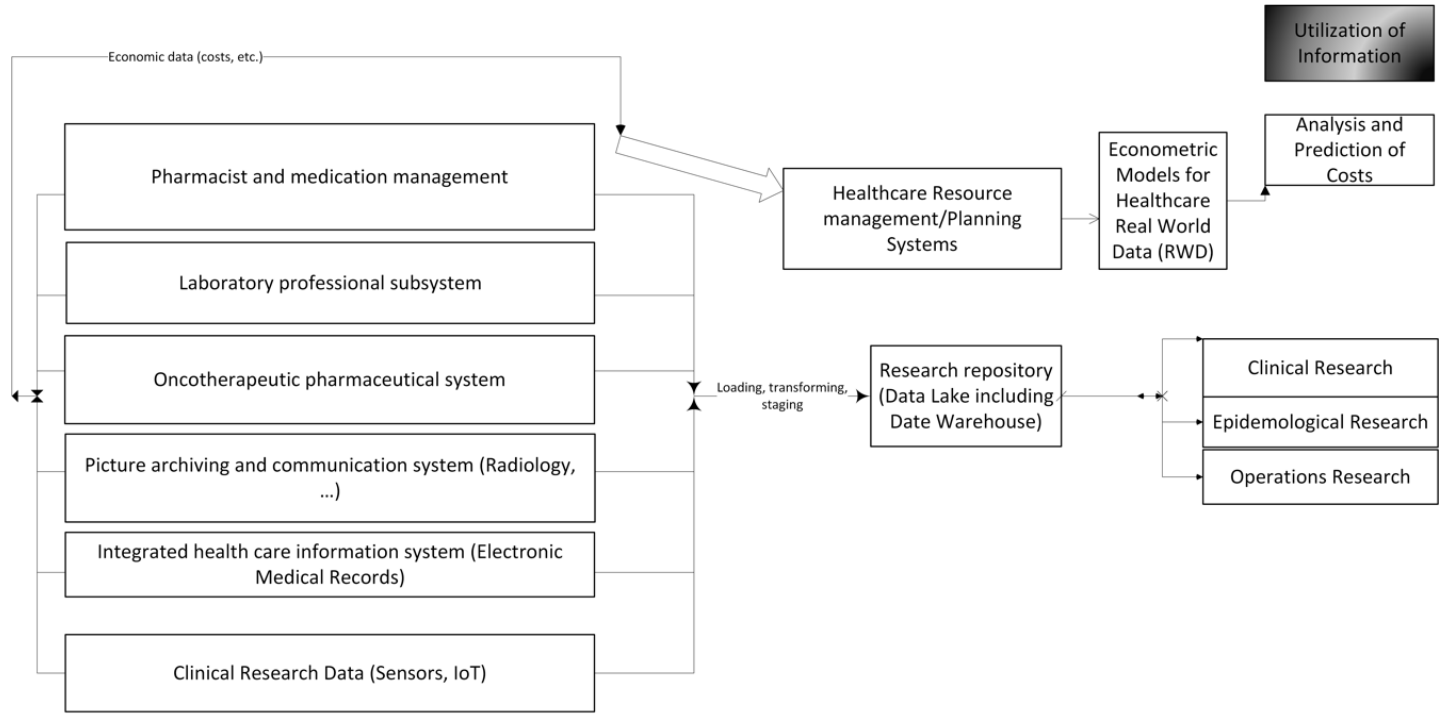

Figure 1. Sources of data and their usage

Generally, it assumed that the data coming from source systems are in good quality however, the market and administrative forces have not enforced a high level of standards of data quality in health care data. The typical life history of data can be seen in Figure 1. On the left part of the diagram, the various major source systems of healthcare data can be found. The data are represented as patients' electronic personal/health/medical records, pharmaceutical events at hospitals/clinics, clinical/diagnostic laboratory data, geo-codes for geographical information, and other loosely coupled data related to management, and business administration.

Our case study deals with a moderate size healthcare environment within a Medical School so that the Vs (volume, velocity, variety, veracity, variability, value) of the Big Data are as follows. A population of a million patients may generate electronic health records in the order of terabytes yearly the clinical images (PACS) could be a magnitude higher. Primarily, the variability of structured, semi-structured, and unstructured data increases the complexity thereby the difficulty of ensuring the single point of truth within the data collection. 
Within a hybrid Data Lake that contains a robust Data Warehouse as well, the life cycle of data commences with transformation, cleansing, and integration. The objective to build up a Data Lake is to separate the daily operation, transactional data from the non-production data collection. Historically, the Data Warehouse technology has been employed for that purpose. During the data staging phase, the data are cleansed and filtered for the target data structure within the Data Warehouse, i.e. the fact table and dimensions. The phase of data staging includes data migration, data integration, translation of codes used for data representation, the transformation between database management systems. The Data Warehouse served as a basis for data analysis traditionally. The ETL (Extract, Transform, Load) procedure is applied for feeding data into the Data Warehouse. During that step the general data cleansing and transformation happens, e.g. amputation of trailing and leading whitespaces, superfluous zeros, standardization of identifiers/identifying numbers, inflicting constraints on data fields, converting English units into metric units.

While the before mentioned data-transformation is carried out relationships among entities may be dropped or harmed. Similar way, the data integration from multiple source systems, can lead to errors that are transmitted into the Data Warehouse.

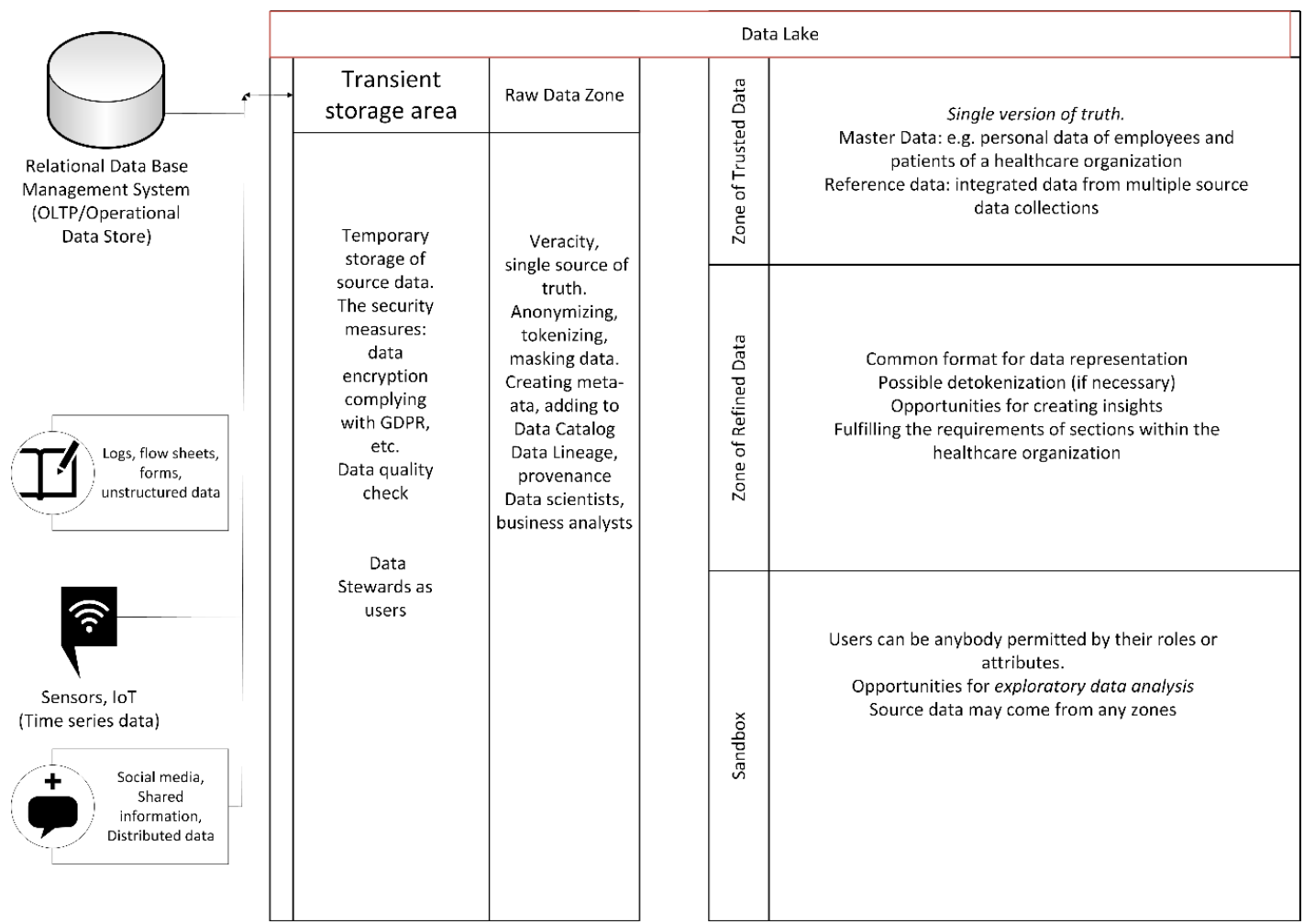

Figure 2. Sources of data and their usage

To overcome the data quality limitations of Data Warehouse, the idea of the Data Lake is conceptualized. The touted idea of Data Lake is that it deposits data in their original form, i.e. the Transient and/or Raw Data zone (see Figure 2; (LaPlante \& Sharma, 2014)) contains the data after an ingestion phase so that the Data lake collects structured data from RDBMS (Relational DataBase Management Systems), semi-structured data (XML, binary XML, JSON, BSON, etc.), and unstructured data along with meta-data that are represented typically in a semi-structured format. The ingestion phase may mean loading, batch processing, data stream processing of source data while the necessary quality checks are carried out utilizing the MapReduce capability (Duggal, et al., 2015). The essential property of the raw data zone is that it is considered as "a single source of truth" as it keeps the data in their original form, however, the masquerading, tokenizing of the data may happen in that zone. Data scientists, business/data analysts can return to that zone when they look for associations and relationships that may have lost during data conversion, transformation, encoding, and encrypting. 
The Trusted Zone executes the procedures for data alteration as quality assurance, compliance with standards, data cleansing, and data validation. In that zone, several data transformations happen to correspond to prescribed local and global policies whereby the data can be considered as a "single version of the truth". This zone may contain master data and the fact data that are governed through the data catalog that is filled in by meta-data automatically or semi-automatically. The data in the Refined Zone undergo several further changes that aim at the usability of data in algorithms of Data Science. These transformations include formatting, potential detokenization, data quality control to fulfill of requirements of algorithms so that models of the subject area (e.g. healthcare) and data analytics can be created. Thereby, knowledge discovery exercises can be carried out and understanding of data collections may be achieved. The access rights of users within each zone should be strictly maintained in the form of Role-Based Access Control, and for the temporary deviations from the baseline can be solved by Attribute-Based Access Rights.

For researchers, managers, and other subject area experts who would like to pursue exploratory data analytics (Myatt, 2007), the Sandbox provides opportunities to set up models, discover associations and relationships among attributes without involving members of IT department and other extra costs. The researcher can bring data into the Sandbox from any other zones within a controlled environment. It is even allowed to export interesting results back to the raw data zone for re-use.

The purpose of the Data Lake and Data Warehouse dedicated to research within e-Health Informatics is to lay the foundation of scientific workflows. The architecture should support several requirements as follows: (1) achieving processing speed through adjusting configuration parameters, (2) exploitation the distribution of data among cluster nodes, (3) usage of provenance data, (4) data placement and scheduling algorithm for input data to prepare the efficient data processing.

\section{CONCLUSION}

As a result of our researches our the proposed Data Lake architecture consists of the above mentioned data zones and a Data Warehouse as well, to support or the information requirements and the workflows of the organization.

\section{ACKNOWLEDGMENT}

The research was supported by the project No. 2019-1.3.1-KK-2019-00011 financed by the National Research, Development and Innovation Fund of Hungary under the Establishment of Competence Centers, Development of Research Infrastructure Programme funding scheme.

\section{REFERENCES}

Duggal, R., Khatri, S. K., \& Shukla, B. (2015). Improving patient matching: single patient view for Clinical Decision Support using Big Data analytics. In B. S. Kapur (Ed.), 4th International Conference on Reliability, Infocom Technologies and Optimization (ICRITO)(Trends and Future Directions) (pp. 1-6). Amity University Uttar Pradesh, Noida, India: IEEE.

LaPlante, A., \& Sharma, B. (2014). Architecting Data Lakes. O’Reilly Media Sebastopol.

Molnár, B., \& Tarcsi, Á. (2013). Design and architectural issues of contemporary web-based information systems. Mediterr. J. Comput. Netw, 9, 20-28.

Myatt, G. J. (2007). Making sense of data: a practical guide to exploratory data analysis and data mining. John Wiley $\&$ Sons.

Shachak, A., \& Reis, S. (2011). The Computer-Assisted Patient Consultation. In Clinical Technologies: Concepts, Methodologies, Tools and Applications (old.: 160-171). IGI Global. 